\title{
Chromosome Segregation Defects Contribute to Aneuploidy in Normal Neural Progenitor Cells
}

\author{
Amy H. Yang, ${ }^{1,4}$ Dhruv Kaushal, ${ }^{2,4}$ Stevens K. Rehen, ${ }^{4}$ Kristin Kriedt, ${ }^{3}$ Marcy A. Kingsbury, ${ }^{4}$ Michael J. McConnell, ${ }^{1,4}$ \\ and Jerold Chun ${ }^{1,2,3,4}$ \\ ${ }^{1}$ Biomedical Sciences and ${ }^{2}$ Neurosciences Graduate Programs and ${ }^{3}$ Department of Pharmacology, School of Medicine, University of California, San Diego, \\ California 92093, and ${ }^{4}$ Department of Molecular Biology, The Scripps Research Institute, La Jolla, California 92037
}

\begin{abstract}
Recent studies based predominantly on nucleotide hybridization techniques have identified aneuploid neurons and glia in the normal brain. To substantiate these findings and address how neural aneuploidy arises, we examined individual neural progenitor cells (NPCs) undergoing mitosis. Here we report the identification of chromosomal segregation defects in normal NPCs of the mouse cerebral cortex. Immunofluorescence in fixed tissue sections revealed the presence of supernumerary centrosomes and lagging chromosomes among mitotic NPCs. The extent of aneuploidy followed the prevalence of supernumerary centrosomes within distinct cell populations. Realtime imaging of live NPCs revealed lagging chromosomes and multipolar divisions. NPCs undergoing nondisjunction were also observed, along with interphase cells that harbored micronuclei or multiple nuclei, consistent with unbalanced nuclear division. These data independently confirm the presence of aneuploid NPCs and demonstrate the occurrence of mitotic segregation defects in normal cells that can mechanistically account for aneuploidy in the CNS.
\end{abstract}

Key words: cortex; mitosis; stem cells; mosaicism; cell death; neurogenesis

\section{Introduction}

During cortical neurogenesis, postmitotic neurons arise from embryonic neural progenitor cells (NPCs), which reside in the ventricular zone (VZ), a proliferative region lining the lateral ventricles of the cerebral hemispheres. Recent analyses of NPC genomes in the developing cerebral cortex primarily by nucleotide hybridization techniques revealed the surprising finding that $\sim 33 \%$ of these cells were aneuploid (Rehen et al., 2001). Although many of the aneuploid NPCs were hypothesized to undergo programmed cell death, a fate common for this population (Blaschke et al., 1996; Kuida et al., 1996; Pompeiano et al., 2000), a portion appeared to survive as neurons and glia during postnatal development and into adult life (Rehen et al., 2001; Kaushal et al., 2003). The mechanisms through which aneuploid neural cells could be generated are not known, but previous work, particularly on neoplastic cells, has identified mitotic chromosome segregation defects as major contributors to cellular aneuploidy (Lengauer et al., 1998; Pihan and Doxsey, 1999).

Mitosis usually ensures equal segregation of chromosomes

Received Aug. 21, 2003; revised Sept. 25, 2003; accepted Sept. 25, 2003.

This work was supported by the National Institute of Mental Health and the Helen L. Dorris Institute for the Study of Neurological and Psychiatric Disorders of Children and Adolescents (J.C.), a predoctoral fellowship from the Pharmaceutical Research and Manufacturers of America Foundation (D.K.), a National Institute of General Medical Sciences Pharmacology training grant (M.J.M. and A.H.Y.), a postdoctoral fellowship from the PEW Latin American Fellows in the Biomedical Sciences (S.K.R.), and a Neuroplasticity of Aging training grant (M.A.K.). We thank Drs. D. Cleveland and S. Dowdy for helpful comments and discussion; C. Higgins and Drs. B. Anliker, J. J. Contos, and J. A. Weiner for critical reading of this manuscript; $M$. Fontanoz and $G$. Kennedy for excellent technical assistance; and $S$. McMullen and J. Sherman for assistance with deconvolution microscopy and live-cell imaging at the University of California, San Diego, Cancer Center Digital Imaging Shared Resource.

Correspondence should be addressed to Dr. Jerold Chun, Department of Molecular Biology, The Scripps Research Institute, 10550 North Torrey Pines Road, ICND118, La Jolla, CA 92037. E-mail: jchun@scripps.edu.

Copyright $\odot 2003$ Society for Neuroscience $\quad$ 0270-6474/03/2310454-09\$15.00/0
(Fig. 1A). One common mechanism for neoplastic aneuploidy is missegregation of lagging chromosomes, or laggards (Saunders et al., 2000; Kirsch-Volders et al., 2002). Laggards are displaced mitotic chromosomes that frequently become encapsulated in a micronucleus and excluded from the daughter nuclei as mitosis ends (Fig. $1 B$ ). As a result, one or both of these daughter nuclei contain less than the euploid number of chromosomes, a condition termed hypoploidy. Thus, laggards and micronuclei are indicators of chromosome missegregation and aneuploidy.

A second mechanism for neoplastic aneuploidy is supernumerary centrosomes (for review, see Pihan and Doxsey, 1999; Brinkley, 2001). During normal mitosis (Fig. 1A), the two centrosomes in a cell nucleate mitotic microtubules and form bipolar spindle poles. Each mitotic chromosome is connected via microtubules to opposite spindle poles to ensure equal bipolar segregation of sister chromatids. In cells with supernumerary centrosomes, multipolar spindle poles may direct chromosomes into $>2$ nuclei, resulting in aneuploid progeny with either single or multiple nuclei (Fig. 1C). Alternatively, supernumerary centrosomes may coalesce into two spindle poles to mediate a subtly irregular bipolar division (Pihan and Doxsey, 1999; Brinkley, 2001) that can give rise to unequal segregation or laggards (Fig. $1 D)$. Supernumerary centrosomes also become transmitted to the progeny (Fig. 1C,D) to perpetuate future rounds of aberrant mitoses.

A third mechanism for neoplastic aneuploidy, mitotic nondisjunction, occurs when a metaphase chromosome fails to disjoin and both sister chromatids move to one of the daughter cells (Fig. $1 E$ ) (Kirsch-Volders et al., 2002). As a result, one daughter cell receives one copy of a chromosome (becoming monosomic) and the other daughter cell receives three copies (becoming trisomic). 
A

Normal Mitosis

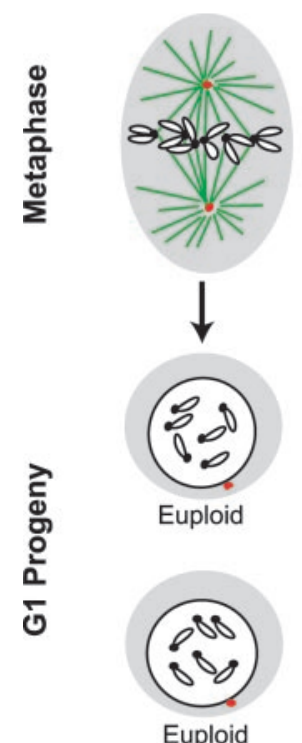

Euploid
B

\section{Lagging chromosomes}

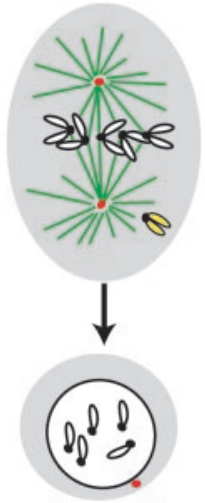

Hypoploid

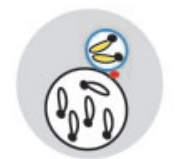

Hypoploid

$+$

Micronucleus
C

\section{Supernumerary centrosomes: multipolar division}
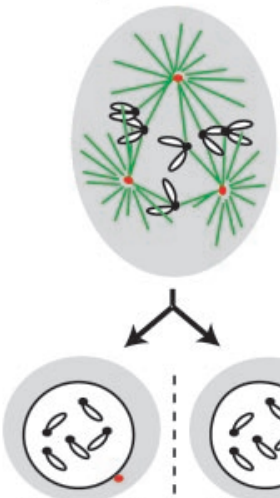

Hypoploid

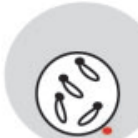

Hypoploid

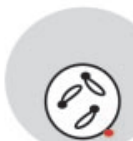

Hypoploid
D

\section{Supernumerary centrosomes: bipolar division}

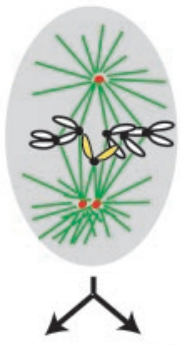

E

\section{Non-disjunction}
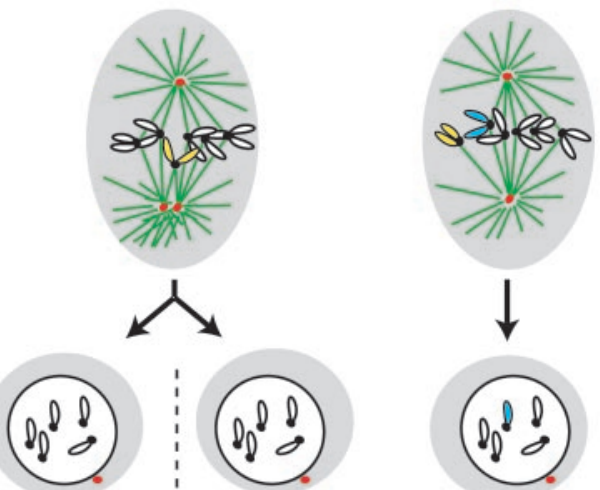

Hypoploid
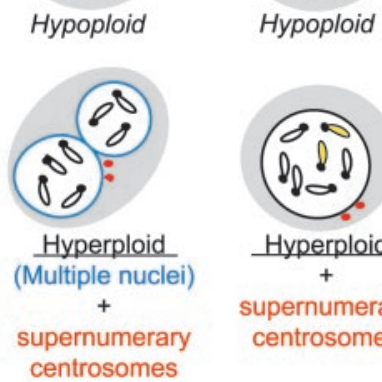

Hyperploid

$+$

supernumerary centrosomes

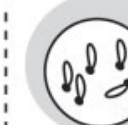

Hypoploid

(monosomic)

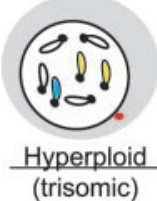

Micronucleus

$+$

supernumerary centrosomes

\begin{tabular}{|c|c|c|c|c|}
\hline$\bullet$ & $\begin{array}{l}\text { Centrosome/ } \\
\text { Spindle pole }\end{array}$ & Metaphase chromosome & $\begin{array}{l}\text { Centromere/ } \\
\text { Kinetochore }\end{array}$ & Micronucleus \\
\hline & Microtubule & Daughter chromosome & Nucleus & Multiple nuclei \\
\hline
\end{tabular}

Figure 1. Chromosome segregation defects can lead to aneuploidy. $A$, During normal mitosis, metaphase chromosomes establish bipolar attachment via microtubules to the spindle poles, align at the metaphase plate, and segregate equally into two daughter cells. $B-E$, Chromosome segregation defects and consequently aneuploidy can result from lagging chromosomes ( $B$ ), supernumerary centrosomes $(C, D)$ and nondisjunction $(E)$, each depicted here as one of many mitotic configurations possible. Metaphase chromosomes involved in missegregation are yellow $(B, D, E)$, except for $C$, in which all chromosomes are involved in a multipolar division. The second copy of the affected chromosome is blue in $E$ to illustrate distribution of the four daughter chromosomes in the progeny. Gray ovals/circles, Cytoplasm.

Often mitoses involving nondisjunction appear to undergo bipolar divisions (Kirsch-Volders et al., 2002), thus eluding detection by mitotic morphology alone.

The common occurrence of aneuploidy in tumor cells and normal embryonic NPCs raises the possibility that mechanisms responsible for neoplastic aneuploidy may likewise function in the normal CNS. Here we report that lagging chromosomes, supernumerary centrosomes, and nondisjunction contribute to the generation of aneuploidy among NPCs.

\section{Materials and Methods}

Cell preparation. All animal protocols have been approved by the Animal Subjects Committee at the University of California, San Diego, and conform to National Institutes of Health guidelines and public law. TR cells, an immortalized NPC line, were cultured as described previously (Chun and Jaenisch, 1996). BALB/c mice (Charles River Laboratories, Wilmington, MA) were used for the isolation of lymphocytes and embryonic NPCs. To prepare adult lymphocytes, splenocytes from spleens of female mice were cultured in RPMI 1640 (Invitrogen, Carlsbad, CA) supplemented with $10 \mu \mathrm{g} / \mathrm{ml}$ phytohemagglutinin (Sigma Aldrich, St. Louis, MO), $10 \%$ fetal calf serum (FCS; Hyclone, Logan, UT) and 1\% penicillin-streptomycin for $36-48 \mathrm{hr}$ at $37^{\circ} \mathrm{C}$; nonadherent cells were harvested for analysis. To prepare acutely isolated NPCs, timed-pregnant females were killed and the embryos were removed at embryonic day 12 (E12) and E14; cerebral cortices were dissected and dissociated immediately.
To examine nondisjunction in NPCs, intact cortical hemispheres were cultured in Opti-MEM I (Invitrogen) supplemented with $6 \mu \mathrm{g} / \mathrm{ml}$ cytochalasin B (Sigma Aldrich), 2.5\% FCS, $20 \mathrm{~mm}$ D-glucose, $55 \mu \mathrm{M}$ $\beta$-mercaptoethanol, and $1 \%$ penicillin-streptomycin for $20 \mathrm{hr}$ at $37^{\circ} \mathrm{C}$ with gentle agitation, followed by dissociation to yield isolated cells. For all cell types, $\sim 1 \times 10^{6}$ cells were seeded onto chamber slides (Nunc, Naperville, IL) precoated with Cell-Tak $\left(2 \mathrm{mg} / \mathrm{cm}^{2}\right.$; Becton Dickson, Franklin Lakes, NJ) and allowed to settle for $30 \mathrm{~min}$ at room temperature, or $37^{\circ} \mathrm{C}$.

Fixation and immunofluorescence. Acutely isolated cells were fixed in $4 \%$ paraformaldehyde for $10 \mathrm{~min}$ at room temperature, and rinsed three times for $10 \mathrm{~min}$ each in PBS. BALB/c embryos were fixed in $4 \%$ paraformaldehyde at $4^{\circ} \mathrm{C}$ overnight, cryoprotected by equilibrating in increasing concentrations of sucrose solution, embedded in Tissue-Tek OCT (Optimal Cutting Temperature; Miles Inc., Elkhart, IN) and quickly frozen on powdered dry ice. Sagittal sections were cut at 10-14 $\mu \mathrm{m}$ on a Frigocut 2800 E cryostat (Leica, Nussloch, Germany) and collected onto electrostatically charged slides (Superfrost Plus; Fisher Scientific, Pittsburgh, PA). The following steps for immunofluorescence were performed at room temperature. Cells or cortical sections were blocked in $1 \%$ bovine serum albumin and $0.5 \%$ Triton X-100 in PBS for $1 \mathrm{hr}$. Primary antibody dilutions were determined empirically, prepared in the blocking solution, and applied to slides. The primary antibodies used in this study were anti-phosphorylated vimentin (Medical and Biological Laboratories, Nagoya, Japan), anti-phosphorylated histone H3 (Upstate 
Biotechnology, Lake Placid, NY), anti-pericentrin (BabCO, Richmond, $\mathrm{CA}$ ), anti- $\gamma$-tubulin (Sigma Aldrich), anti- $\alpha$-tubulin (Cytoskeleton, Denver, CO), and anti-nestin (PharMingen, San Diego, CA) antibodies. After overnight incubation, slides were rinsed three times for $10 \mathrm{~min}$ each in PBS and incubated with FITC- or Cy3-conjugated secondary IgGs (Chemicon, Temecula, CA) for $1 \mathrm{hr}$. The slides were then stained with 4',6-diamidino-2-phenylindole (DAPI, $0.3 \mu \mathrm{g} / \mathrm{ml}$; Sigma Aldrich) and coverslipped with Vectashield (Vector Laboratories, Burlingame, CA).

Metaphase chromosome spread analysis by spectral karyotyping. The analysis was performed as described previously (Rehen et al., 2001). Briefly, intact embryonic cortical hemispheres were cultured in OptiMEM I supplemented with $100 \mathrm{ng} / \mathrm{ml}$ colcemid (Invitrogen), 2.5\% FCS, $20 \mathrm{~mm}$ D-glucose, $55 \mu \mathrm{M} \beta$-mercaptoethanol, and $1 \%$ penicillin-streptomycin at $37^{\circ} \mathrm{C}$, with gentle agitation for $3 \mathrm{hr}$. The hemispheres were dissociated; cells were subjected to hypotonic swelling (by incubating in $75 \mathrm{~mm} \mathrm{KCl}$ ) and fixed in methanol:acetic acid (3:1). Chromosome spreads were prepared on clean, dry slides (Fisher Scientific) following standard protocols (Barch et al., 1997). Slides were prepared for spectral karyotyping (SKY) or DAPI staining alone per manufacturer's instructions [Applied Spectral Imaging (ASI), Carlsbad, CA]. Karyotypes were determined from micrographs captured using a $100 \times$ [numerical aperture (NA), 1.4] oil-immersion lens on a Zeiss Axioplan2 microscope (Carl Zeiss Microimaging, Thornwood, NY), Spectracube interferometer and cooled CCD camera (ASI), and Spectral Imaging and SKYview software (ASI).

Fluorescence in situ hybridization. Isolated NPCs on slides were fixed in methanol:acetic acid $(3: 1)$ at $4^{\circ} \mathrm{C}$ for overnight or longer. Slides were rehydrated in $2 \times$ SSC for $10 \mathrm{~min}$ at room temperature, dehydrated in ethanol, and air dried. Whole chromosome paints from chromosomes 4 and $\mathrm{X}$ ( $5 \mu \mathrm{l}$ of each paint per slide; ASI) were denatured at $74^{\circ} \mathrm{C}$ for 10 min, followed by renaturation at $37^{\circ} \mathrm{C}$ for 60 min. The chromosome paint mixture was immediately applied to slides, coverslipped, and denatured at $74^{\circ} \mathrm{C}$ for $5 \mathrm{~min}$ on a slide warmer. After sealing the edges of the coverslip, the slide was incubated in a humidified chamber at $37^{\circ} \mathrm{C}$ overnight. Two washes of $2 \times$ SSC ( 10 min each) followed by two washes of $0.1 \times$ SSC $(10 \mathrm{~min}$ each) were applied to the slide at $65^{\circ} \mathrm{C}$. DAPI was added to the first $0.1 \times$ SSC wash to a final concentration of $0.3 \mu \mathrm{g} / \mathrm{ml}$. The slide was rinsed in $4 \times$ SSC $/ 0.1 \%$ Tween 20 for $5 \mathrm{~min}$ at room temperature, partially air dried, and coverslipped with Vectashield.

Image acquisition of fixed samples. Deconvolution microscopy of mitotic NPCs in embryonic cortical sections (see Fig. $3 B, C$ ) were captured with a DeltaVision (Applied Precision, Issaquah, WA) imaging station: an inverted epifluorescence microscope (Nikon TE-200; Nikon, Tokyo, Japan) with a $60 \times(\mathrm{NA}, 1.4)$ oilimmersion lens, and a Photometrics cooled CCD camera. Approximately 60-80 optical sections spaced by $0.3 \mu \mathrm{m}$ were taken. Exposure times were set such that the camera response was in the linear range for each fluorophore. The data sets were deconvolved and analyzed using SoftWoRx software (Applied Precision) on a Silicon Graphics (Mountain View, CA) Octane workstation. Maximal projection volume views are shown. Other images were captured using a $40 \times(\mathrm{NA}, 0.75)$ or a $100 \times(\mathrm{NA}, 1.4)$ oil-immersion lens on a Zeiss Axioplan2 microscope with a CCD camera (ASI) and EasyFish software (ASI). Images were prepared in Photoshop (Adobe Systems, Mountain View, CA).

Analysis of mitotic progression and chromosome dynamics in live NPCs. Cerebral cortices from E12-E15 BALB/c embryos were dissected 3 and $10(38, X Y,-3,-10)$. and the meninges were carefully removed. Cortical clusters were generated by triturating tissues eight to nine times in culture medium: OptiMEM I supplemented with $10 \mathrm{ng} / \mathrm{ml}$ fibroblast growth factor-2 (FGF-2; Invitrogen), $20 \mathrm{ng} / \mathrm{ml}$ epidermal growth factor (EGF; Invitrogen), $2.5 \%$ FCS, $20 \mathrm{~mm}$ D-glucose, $55 \mu \mathrm{M} \beta$-mercaptoethanol, and $1 \%$ penicillinstreptomycin. The cortical clusters were then plated on the center of a 40 $\mathrm{mm}$ coverslip pretreated with Cell-Tak and incubated for 20-24 hr at $37^{\circ} \mathrm{C}$. Two hours before imaging, the coverslip was mounted onto a closed, live-cell micro-observation system (FCS2; Bioptechs, Butler, PA) set at $35-37^{\circ} \mathrm{C}$ and perfused with the culture media supplemented with $200 \mathrm{~nm}$ of cell-permeant nucleic acid dye Syto-16 (Molecular Probes, Eugene, OR). Images were captured with the DeltaVision deconvolution microscope system. In general, six to seven optical sections spaced by 2 $\mu \mathrm{m}$ were taken by a $40 \times(\mathrm{NA}, 1.3)$ oil-immersion lens every $10 \mathrm{~min}$ for 4-8 hr. At each optical plane, a fluorescence image and differential interference contrast image were taken. Maximal projection volume views of deconvolved images are shown. Images were prepared in Photoshop.

Scoring of nuclear abnormalities. A minor nucleus $\leq 50 \%$ of the main nuclear diameter was scored as a micronucleus. A cell with more than one nucleus whose sizes did not differ by $>50 \%$ were scored as having multiple nuclei.

\section{Results}

\section{Lagging chromosomes in mitotic NPCs}

NPC mitoses occur along the ventricular surface of the VZ (Seymour and Berry, 1975). To visualize mitotic NPCs and their chromosomes, sections of mouse embryonic cerebral cortices were immunolabeled for phosphorylated histone H3 (phospho-H3), which identifies condensed chromosomes (Hendzel et al., 1997), and for phosphorylated vimentin (phospho-vimentin), a cyto-

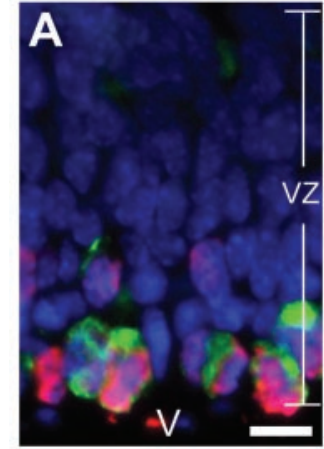

E

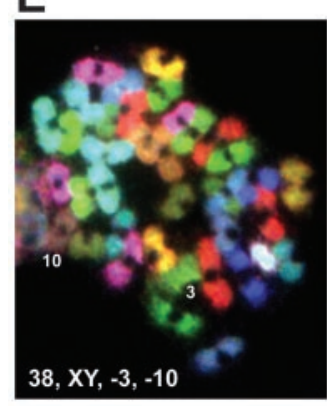

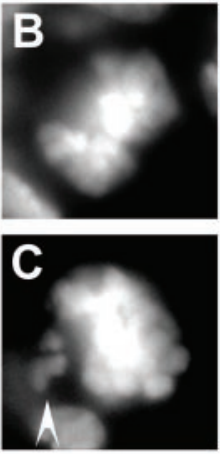

$\mathbf{F}$

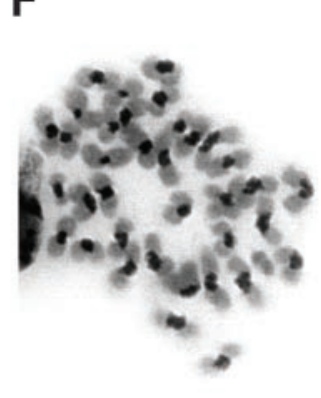

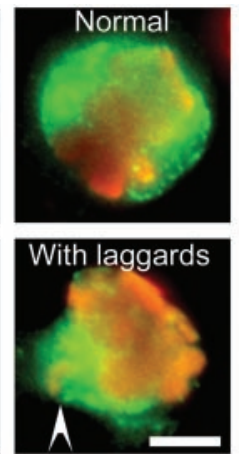

G
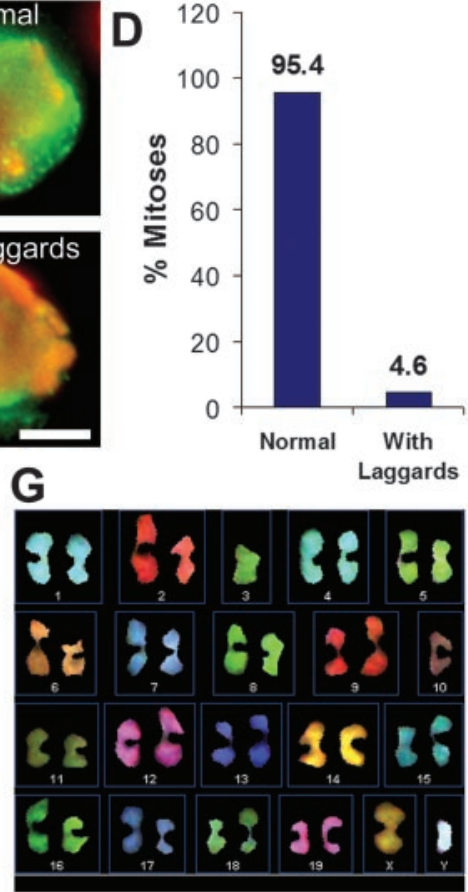

Figure 2. NPCs have lagging chromosomes and aneuploidy. A, Micrograph of the embryonic cerebral cortex. Immunofluorescence for the mitotic chromosome marker phospho-H3 (red) and the mitotic NPC marker phospho-vimentin (green) identifies mitotic NPCs along the ventricular surface of the VZ. Nuclei are counterstained with DAPI. V, Ventricle. Scale bar, $10 \mu \mathrm{m} . B, C$, High-magnification micrographs of mitotic NPCs. Left panels show DAPI-stained nuclei/chromosomes; right panels show the same cells with phospho-H3-positive chromosomes surrounded by phospho-vimentin labeling. Some mitoses appear morphologically normal ( $B)$; others exhibit lagging chromosomes ( $C$; arrowhead). Scale bar, $5 \mu \mathrm{m}$. D, Percentage of mitotic cells $(n=$ 300 ) colabeled for both phospho-H3 and phospho-vimentin containing lagging chromosomes. $E-G, S K Y$ analysis of a representative hypoploid chromosome spread prepared from NPCs. Spectral image ( $E$ ), inverse DAPI image ( $F$ ), and karyotype table $(G)$ are shown. The euploid chromosome number for Mus musculus is 40. The chromosome spread has only one copy of chromosomes 

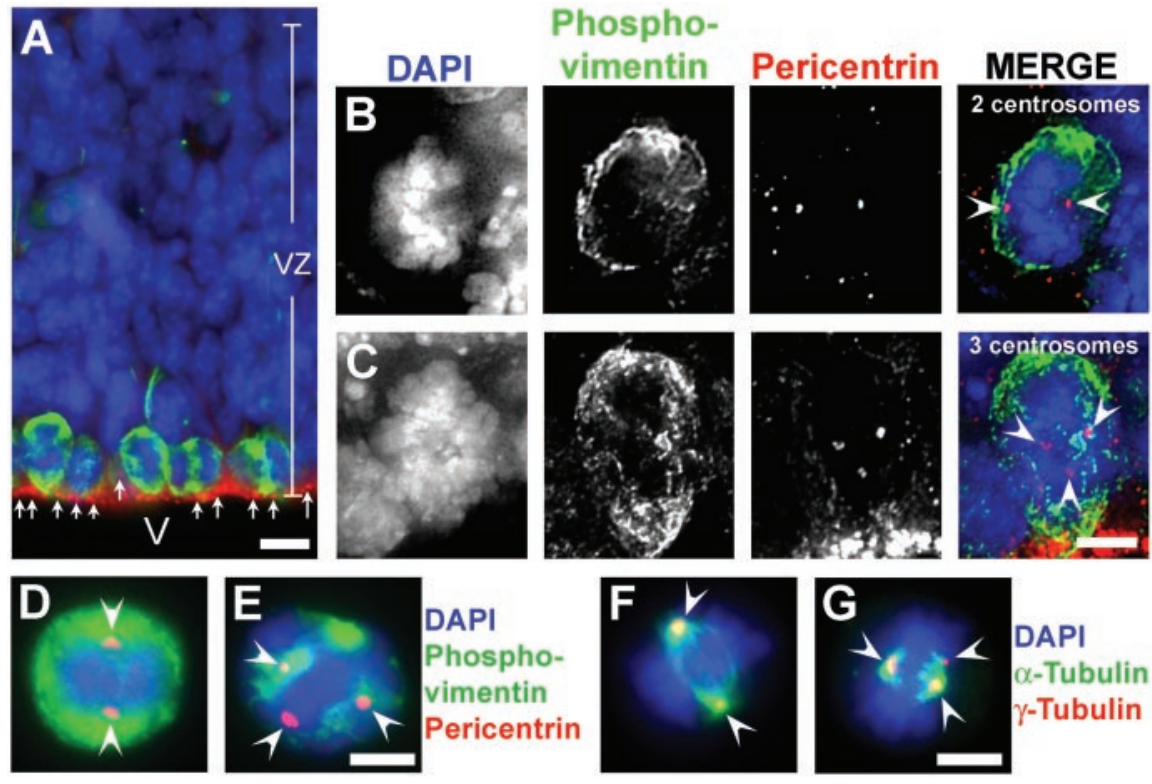

Figure 3. Mitotic NPCs harbor supernumerary centrosomes. $A-C$, Supernumerary centrosomes in situ. $A$, Micrograph of the embryonic cerebral cortex with immunofluorescence for phospho-vimentin (green), a mitotic NPC marker, and pericentrin (red), a centrosome marker. The polarized distribution of centrosomes along the ventricular surface of the VZ is illustrated. Nuclei are counterstained with DAPI. B, C, High-magnification, maximal projection views from deconvolution microscopy of mitotic NPCs double-labeled for phospho-vimentin and pericentrin. A typical prometaphase/metaphase NPC has two centrosomes that act as bipolar spindle poles $(B)$. However, some mitotic NPCs contain supernumerary $(>2)$ centrosomes $(C)$. Scale bars: $A, 10 \mu \mathrm{m} ; C, 5$ $\mu \mathrm{m} . D-G$, Supernumerary centrosomes in acutely isolated NPCS. $D, E$, Immunofluorescence for phospho-vimentin (green) and pericentrin (red). $F, G$, Immunofluorescence for $\alpha$-tubulin (microtubule marker; green) and $\gamma$-tubulin (centrosome marker; red). Mitoses with two centrosomes $(D, F)$ and mitoses with supernumerary centrosomes $(E, G)$ are shown. Scale bars: $E, G, 5 \mu \mathrm{m}$. Arrows $(A)$ and arrowheads $(B-G)$, Centrosomes.

plasmic marker for mitotic NPCs (Kamei et al., 1998; Noctor et al., 2002) (Fig. 2A). Although the majority of NPC mitoses in the VZ appeared morphologically normal (Fig. $2 B, D$ ), 4.6\% of them harbored lagging chromosomes (Fig. 2C,D). Consistent with this finding, metaphase chromosome spreads analyzed by SKY (Fig. $2 E-G)$, a technique that identifies each chromosome by a unique color, revealed the presence of aneuploid NPCs.

\section{Supernumerary centrosomes in mitotic NPCs}

To determine NPC centrosome number, the centrosome components pericentrin (Doxsey et al., 1994) and $\gamma$-tubulin (Stearns et al., 1991; Zheng et al., 1991) were immunolocalized in embryonic cerebral cortices. Centrosomes in VZ cells were localized to the ventricular surface (Fig. 3A), consistent with previous observations (Meininger and Binet, 1988; Chenn et al., 1998). To focus on mitotic centrosomes, NPCs were double-labeled for pericentrin and phospho-vimentin in the same sections (Fig. $3 A-C$ ). The cytoplasmic distribution of phospho-vimentin facilitated delineation of cell boundaries and allowed enhanced visualization of centrosomes in mitotic NPCs, using three-dimensional reconstruction by deconvolution microscopy (Fig. 3B,C). In addition to morphologically normal mitotic NPCs with two centrosomes (Fig. $3 B$ ), variations in centrosome number were also identified (three centrosomes for the cell in Fig. 3C).

The quantitation of mitotic NPCs with supernumerary centrosomes is inherently difficult in situ, because of the high cell density and enrichment of both interphase and mitotic centrosomes at the ventricular surface. Immunofluorescent detection of pericentrin and phospho-vimentin (Fig. 3D), or $\gamma$-tubulin and $\alpha$-tubulin (a marker for microtubules) (Fig. $3 F$ ) in acutely isolated cells revealed that $96.8 \%$ of mitotic NPCs had the expected
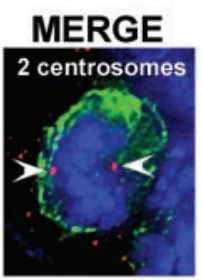

number of centrosomes. However, 3.2\% of mitotic NPCs harbored supernumerary centrosomes (Fig. 3E,G). These results both confirmed the existence of supernumerary centrosomes among mitotic NPCs in situ, and demonstrated the ability of supernumerary centrosomes to nucleate mitotic microtubules and hence, form functional spindle poles (Fig. 3G).

\section{Correlation between supernumerary centrosomes and aneuploidy}

In many neoplasms, numerical centrosome abnormalities are thought to play a role in genomic instability. Supernumerary centrosomes and genomic instability have been reported to occur in aneuploid, but not diploid, tumor cell lines (Ghadimi et al., 2000). Moreover, experimentally induced supernumerary centrosomes in near-diploid cells lead to aneuploidy/ polyploidy (Pihan et al., 2001).

To determine the relationship between supernumerary centrosomes and aneuploidy, an immortalized NPC line, "TR" (Chun and Jaenisch, 1996), was introduced as a model of NPCs that have enhanced levels of supernumerary centrosomes. The TR line displays many characteristics of NPCs (Chun and Jaenisch, 1996; Fukushima et al., 2002), including a fusiform/bipolar morphology (Fig. 4A). TR cells were derived from embryonic NPCs by retroviral transduction of SV40 large $\mathrm{T}$ antigen and vras (Chun and Jaenisch, 1996), which may induce supernumerary centrosomes associated with genomic instability (Levine et al., 1991; Saavedra et al., 1999). Indeed, compared with adult lymphocytes, a cytogenetic standard (Barch et al., 1997), a strong linear correlation $\left(R^{2}>0.99\right)$ was detected between the percentage of mitoses with supernumerary centrosomes and the extent of aneuploidy (Fig. 4D). TR cells presented supernumerary centrosomes (Fig. 4B,C) at a level of $8.9 \%$, relative to $3.2 \%$ in NPCs and $0.7 \%$ in lymphocytes (Fig. $4 D$ ). Correspondingly, the extent of aneuploidy was highest in TR cells (85.7\%) (Fig. 4E), in contrast with $33.2 \%$ in NPCs (Fig. $4 E$ ) and $3.4 \%$ in lymphocytes (Fig. 4D). The tetraploidy-inducing effect of large T antigen (Levine et al., 1991) may explain the existence of a tetraploid population in TR cells (Fig. 4E). The preponderance of TR cells with less-thantetraploid chromosome content is thus consistent with the expected action of supernumerary centrosomes. Together, the percentage of mitoses with supernumerary centrosomes and the extent of aneuploidy appear to correlate positively with each other in a model of NPCs.

\section{Time-lapse imaging of lagging chromosomes and} multipolar spindles

Lagging chromosomes and supernumerary centrosomes observed in fixed NPCs represent plausible mechanisms for NPC aneuploidy. To understand the dynamics of the mitotic machinery as a source of aneuploidy among NPCs, time-lapse videomicroscopy was used. Clusters of embryonic cortical cells were cultured for 20-24 hr in the presence of EGF and FGF-2, similar to established protocols for expanding NPCs (Svendsen et al., 1998; 
Allen et al., 2001). Immunofluorescence revealed that all the phospho-H3-positive mitotic cells examined also expressed nestin, an intermediate filament protein found in NPCs (data not shown). To enable visualization of chromosome behavior during mitosis, the cluster cultures were incubated with a cell-permeant green fluorescent DNA dye, Syto-16.

The majority $(93.4 \%$; $n=76)$ of examined NPC mitoses appeared normal, with a mean prometaphase/metaphase duration of $57 \pm 20 \mathrm{~min}$ (mean $\pm \mathrm{SD})$. However, irregular mitotic chromosome movements were also observed in $6.6 \%$ ( 5 of 76 ) of mitoses: two mitoses involved micronucleation, and three involved multipolar spindles. An example of anaphase lagging chromosomes followed by micronucleation is shown in a time-lapse series in Figure 5. This cell entered prometaphase at time 0:00 (data not shown) and persisted in prometaphase/metaphase for an unusually long period (160 min), accompanied by spindle rotations (Fig. $5 A-D$ ) (Adams, 1996; Haydar et al., 2003), before anaphase initiation (Fig. 5E). During late anaphase, lagging chromosomes were visible (Fig. $5 G$, arrow). Subsequently, anaphase-telophase transition (Fig. 5G,H) occurred with normal kinetics, generating a micronucleus (arrow in Fig. 5H,I) encapsulating lagging chromosomes, in addition to two other nuclei of unequal size. This aberrant mitosis generated two daughter cells, one of which contained a micronucleus.

Mitoses involving multipolar spindles were also documented; a representative time-lapse sequence is illustrated in Figure 6. Similar to the mitosis shown in Figure 5, this cell persisted in prometaphase/metaphase for a long period (120 min), with dynamic chromosome movements (Fig. 6A-E). The duration of aberrant mitotic NPCs (Figs. 5, 6) was twofold to threefold longer than bipolar mitoses, suggesting an attempt to align maloriented chromosomes during metaphase delay. In this case, tripolar anaphase (Fig. 6F, G) followed, as revealed by optical sectioning. Chromosomes were segregated into three groups: the chromosome group in the dashed circle (center) was positioned $\sim 4 \mu \mathrm{m}$ above the other circled groups (Fig. $6 F$ ). As the division neared the end, the three nuclei moved away from each other (Fig. 6H,I). This tripolar mitosis resulted in three aneuploid daughter cells in which the replicated chromosomes segregated into three nuclei of approximately equal size.

\section{Nuclear abnormalities in NPCs}

Mitotic chromosome missegregation manifested as lagging chromosomes and multipolar divisions detected in fixed tissue (Figs. 2, 3) and live NPCs (Figs. 5, 6) can lead to the formation of micronuclei and multiple nuclei (Fig. 1). Among fixed interphase cortical cells that were acutely isolated, micronuclei and multiple nuclei were detected in $4.8 \%$ and $2.2 \%$, respectively, of the nestin-positive NPC population (Fig. 7A). The majority of micronuclei in NPCs were positively labeled for centromeres by
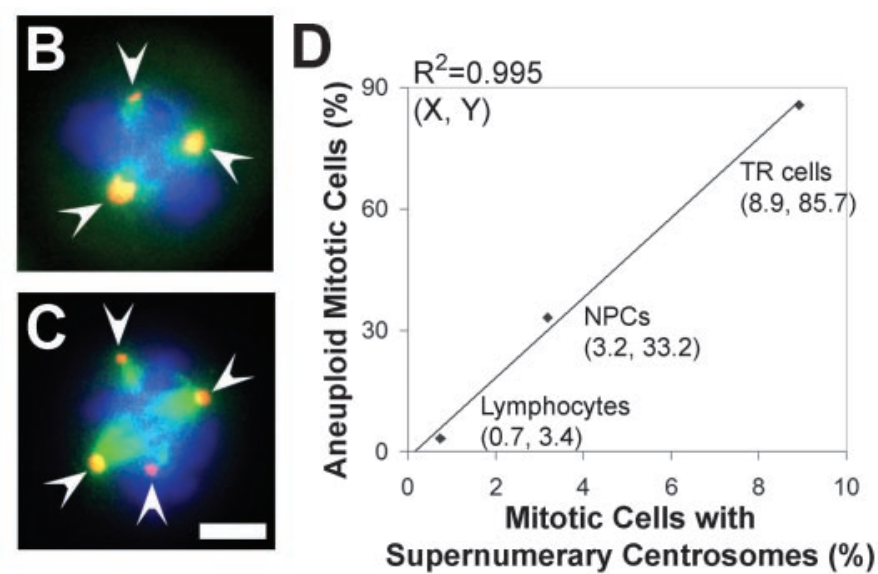

Supernumerary Centrosomes (\%)

NPCs

(33.2\% Aneuploid)

TR cells

(85.7\% Aneuploid)

TR cells with diploid (40)

and tetraploid (80)

chromosome numbers

Figure 4. The extent of aneuploidy correlates positively with the prevalence of supernumerary centrosomes. $A-C$, Immuno(arrowheads) frequently organize multipolar spindles. Nuclei are counterstained with DAPI (blue). Scale bars: $A, 10 \mu \mathrm{m} ; C, 5 \mu \mathrm{m}$. $D$, Linear relationship between percent mitoses with supernumerary centrosomes and extent of aneuploidy for lymphocytes, NPCs, and TR cells. $R^{2}=0.995$. Quantitation of mitoses with supernumerary centrosomes: 134 lymphocytes, 428 NPCs, and 157 least squares linear regression analysis. E, Levels of aneuploidy in NPCs (33.2\%) and TR cells (85.7\%). Black line traces the profile NPC aneuploidy. Gray columns illustrate the chromosome number histogram for TR cells; green columns mark the positions of 40 and 80 chromosomes, which represent diploidy and tetraploidy, respectively, for M. musculus. Data on lymphocyte and NPC aneuploidy in $D$ and $E$ were adapted from Rehen et al. (2001).

pancentromeric fluorescence in situ hybridization (FISH) (data not shown), suggesting the presence of whole chromosomes in these micronuclei (Fenech, 2000). To examine the distribution of individual chromosomes in micronucleated cells, dual-color FISH for chromosomes 4 and $\mathrm{X}$ was applied to acutely isolated embryonic cortical cells. Normal female mononucleate cells in interphase contain two copies of chromosomes 4 and X (Fig. 7B). In a fraction of micronucleated cells, the micronucleus was positively labeled for either or both chromosomes, accompanied by the hypoploid main nucleus (Fig. 7C,D). Together, these data provide evidence that micronucleation in NPCs involves whole chromosomes and are consistent with numerical chromosomal variation in NPCs (Rehen et al., 2001).

\section{Nondisjunction in NPCs}

The simultaneous production of trisomic and monosomic daughter cells in a single round of mitosis is characteristic of a nondisjunction event (Fig. $1 \mathrm{E}$ ). To facilitate analysis of this event, a common assay for nondisjunction uses cytochalasin $\mathrm{B}$, which blocks cytokinesis during mitosis to generate binucleate cells that retain both daughter nuclei in the same cytoplasm (Fenech, 2000; Kirsch-Volders et al., 2002). The cytokinesis-blocked binucleate cells are then hybridized with a chromosome-specific probe to 

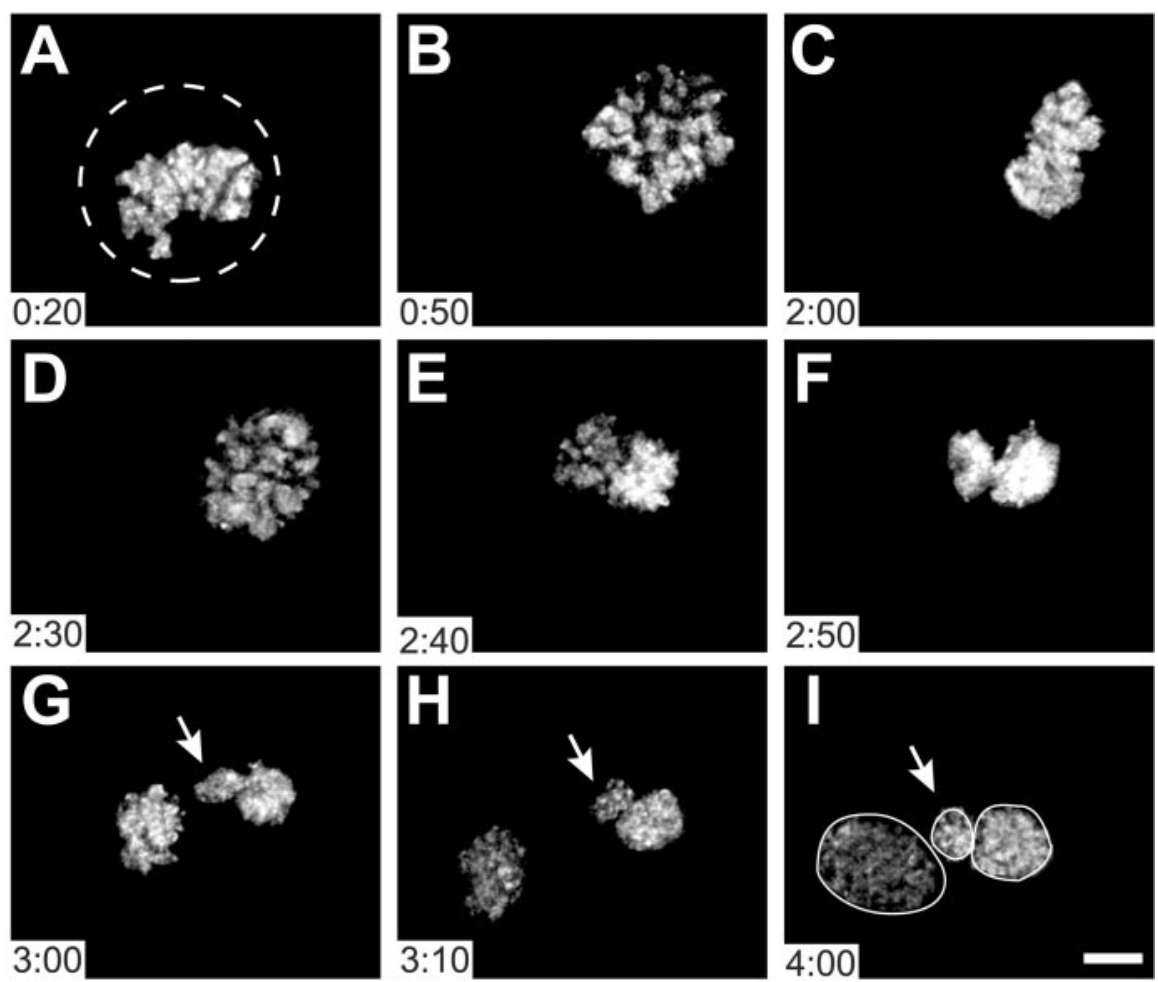

Figure 5. Mitosis with lagging chromosomes in a live NPC leads to micronucleation. $A-D$, Prometaphase/metaphase. The cell enters prometaphase at time 0:00 (denoting hour:minute). Prometaphase/metaphase lasts $160 \mathrm{~min}$, accompanied by spindle rotations. E-G, Anaphase. Lagging chromosomes are visible during late anaphase (G; arrow). H, Telophase. Chromosome decondensation and nuclear envelope reformation take place, generating a micronucleus (arrow) enclosing lagging chromosomes in addition to two other daughter nuclei of unequal size. I, Interphase. Arrow indicates the micronucleus. DNA is vitally stained with the cell-permeant dye Syt0-16. For clarity, mitochondrial DNA labeling has been removed from original images (see supplementa Fig. S1 online, available at www.jneurosci.org). Scale bar, $5 \mu \mathrm{m}$.

analyze distribution of the chromosomes between the two daughter nuclei. A binucleate cell that has undergone normal mitosis would contain two copies of the chromosome in each daughter nucleus (a total of four copies). However, nondisjunction for this chromosome would produce three hybridization signals in one daughter nucleus and one signal in the other.

To investigate whether nondisjunction occurred in the developing brain, intact embryonic cerebral cortices were incubated with cytochalasin B, dissociated, and analyzed by dual-color FISH for chromosomes 4 and X. Because the vast majority of mitotic cells in these preparations were NPCs (Rehen et al., 2001), binucleate cells produced by cytochalasin B exposure were predominantly NPCs. In addition to binucleate NPCs with a normal (2:2) distribution of hybridization signals (Fig. 8A), binucleate NPCs with a 3:1 distribution of chromosome 4 or chromosome X (Fig. $8 B$ ) were detected. Figure $8 C$ shows a binucleate NPC with a 3:1 hybridization pattern for both chromosomes 4 and $\mathrm{X}$, indicating nondisjunction of both chromosomes during the mitosis before cytochalasin B exposure. Assuming that no other chromosomes had also missegregated during this mitosis, two aneuploid daughter cells would have been generated, one trisomic and the other monosomic for chromosomes 4 and X. Segregation patterns of these two chromosomes revealed nondisjunction to be a rare event during cortical neurogenesis, consistent with the scarcity of hyperploidy among aneuploid NPCs.

\section{Discussion}

This study provides the first evidence that mitotic events can mechanistically account for aneuploidy and the production of genetic mosaicism during normal brain development. The occurrence of mitotic errors in NPCs, demonstrated by distinct and complementary techniques, independently confirms the existence of aneuploidy in the nervous system revealed by other, less direct approaches (Rehen et al., 2001; Kaushal et al., 2003). NPCs that undergo chromosome missegregation can foster the production of aneuploid NPCs, complementing euploid NPCs, to create a proliferative founder population that is chromosomally variable. These observed mitotic mechanisms can now be invoked to account for the existence of aneuploid postmitotic neurons (Rehen et al., 2001; Kaushal et al., 2003).

How do these chromosome segregation defects account for the observed patterns of NPC aneuploidy? Lagging chromosomes and supernumerary centrosomes predominantly generate hypoploid cells, whereas nondisjunction in a euploid population produces hypoploid and hyperploid cells in a 1:1 ratio (Fig. 1). The operation of all three mechanisms could account for the preponderance of hypoploidy among aneuploid NPCs (Rehen et al., 2001). Although the overall level of chromosome missegregation is lower than the reported prevalence of NPC aneuploidy (Rehen et al., 2001), this discrepancy could be explained by the following. First, the percentage of NPCs observed with chromosome missegregation at any given time is actually an underestimate of a larger aneuploid NPC pool, because aneuploid progeny generated by a missegregation event can undergo normal bipolar divisions and elude morphological detection. Second, our documentation of mitotic aberrations by videomicroscopy relied heavily on mitotic morphology, and ignored subtle aberrations such as coalescence of supernumerary centrosomes into a bipolar spindle, and nondisjunction involving a single chromosome. Finally, one cannot rule out the possibility that unexplored or new mechanisms may contribute directly to the generation of aneuploidy during cortical neurogenesis. Such explanations are not mutually exclusive, and all might contribute to the actual level of aneuploidy existent in NPCs.

Aneuploidy produced by mechanisms of chromosome missegregation during neurogenesis is reminiscent of the genomic instability present in many tumors. Tumorigenesis is characterized by the opportunistic growth of genetically unstable cells that gain a selective advantage over normally growing cells; neoplastic growth succeeds over alternative fates of nongrowth or cell death (Cahill et al., 1999). Could aneuploid neural cells also undergo a similar form of selection during neurogenesis? Previous studies have identified extensive cell death during cortical development (Blaschke et al., 1996; Kuida et al., 1996; Pompeiano et al., 2000), which is a likely fate of some aneuploid NPCs (Rehen et al., 2001). However, aneuploid neurons and glia are also present in the mature brain (Rehen et al., 2001; Kaushal et al., 2003), suggesting that many aneuploid neural cells survive. A plausible hypothesis is that NPCs may be under an as yet undefined selection pressure (Chun and Schatz, 1999) that, as in tumor cells, may function through genomic changes. We speculate that aneuploidy may 
lead to the death of NPCs harboring deleterious chromosomal combinations, while allowing for survival of NPCs/ newly postmitotic neurons harboring favorable chromosomal complements, including euploidy.

Additional support for the involvement of aneuploidy and genomic instability in neurogenesis and tumorigenesis is found in the multiple molecules involved in DNA repair and surveillance. Many of these molecules are dysregulated in cancer cells (Gao et al., 2000; Khanna and Jackson, 2001; Sharpless et al., 2001; Bergoglio et al., 2002; Lee and McKinnon, 2002), frequently resulting in aneuploidy; intriguingly, the same molecules are also required for normal CNS development. Proteins involved with nonhomologous end joining (XRCC4, DNA ligase IV, Ku70, and Ku80; Barnes et al., 1998; Gao et al., 1998; Frank et al., 2000; Gu et al., 2000), homologous recombination (XRCC2; Deans et al., 2000), base-excision repair (DNA polymerase $\beta$; Sugo et al., 2000), and DNA surveillance molecules p53 (Sah et al., 1995) and Ataxia Telangiectasia Mutated (Allen et al., 2001) have all been implicated in normal CNS development. It is also notable that a high load of endogenous DNA breaks present during neurogenesis (Chun and Schatz, 1999) may predispose NPCs to aneuploidy (Difilippantonio et al., 2000; Griffin et al., 2000; Allen et al., 2001; Bergoglio et al., 2002).

However, parallels between tumorigenesis and neurogenesis diverge in considering cell fate: cancer cells remain proliferative, whereas neurons become postmitotic. Disruption of this normal dichotomy may account for some neuroectodermal brain tumors, which have their origin in proliferative neural cells. For example, many primitive neuroectodermal tumors, which occur with a high incidence in children, exhibit aneuploidy (Bhattacharjee et al., 1997; Weber et al., 1998). Interestingly, the incidence of supernumerary centrosomes correlates positively with aneuploidy in some cerebral tumors (Weber et al., 1998). In contrast, the same form of genomic instability that promotes tumorigenesis likely has fundamentally different consequences for postmitotic neurons, which may tolerate or even benefit from aneuploidy arising during normal development. For example, acquisition of an extra copy of a prosurvival gene (e.g., $b c l-2$ ) (Martinou et al., 1994) might render a neuron less sensitive to cell death.

In conclusion, this study reveals that the mitotic apparatus can mechanistically account for aneuploidy among normal NPCs, and independently supports the finding of CNS chromosomal variation identified by other techniques (Rehen et al., 2001; Kaushal et al., 2003). Because NPCs are descendents of embry-
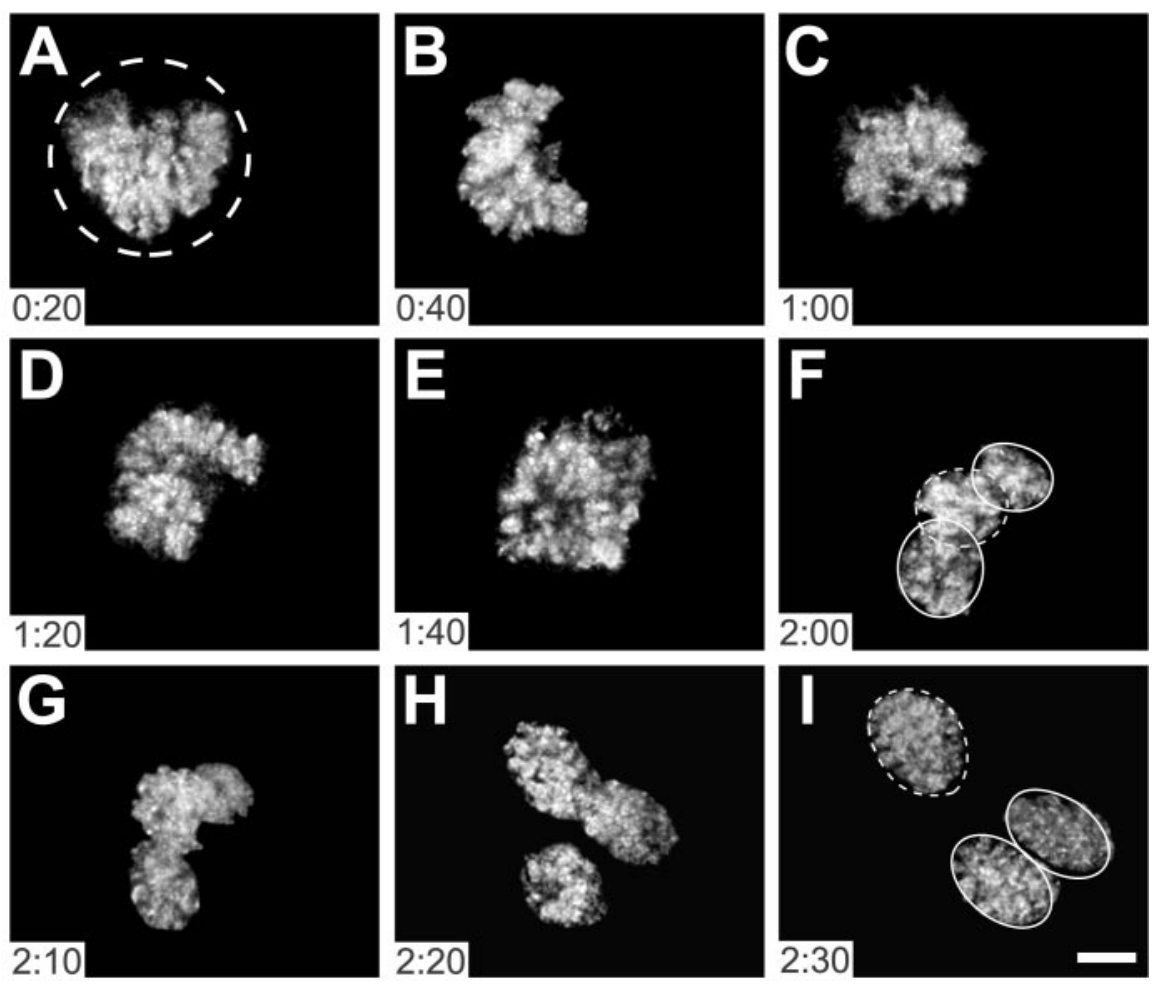

Figure 6. A live NPC undergoes multipolar mitotic division. $A-E$, Prometaphase/metaphase. The cell enters prometaphase at time 0:00. Prometaphase/metaphase lasts $120 \mathrm{~min}$, accompanied by dynamic chromosome movements. $F, G$, Tripolar anaphase. Three segregating chromosome groups arise from this tripolar anaphase: the chromosome group in the dashed circle (center) is positioned $\sim 4 \mu \mathrm{m}$ above the other circled groups $(F)$. $H$, Tripolar telophase. I, Interphase. DNA is vitally stained with the cell-permeant dye Syto-16. For clarity, mitochondrial DNA labeling has been removed from the original images (see supplemental Fig. S2 online, available at www.jneurosci.org). Scale bar, $5 \mu \mathrm{m}$.
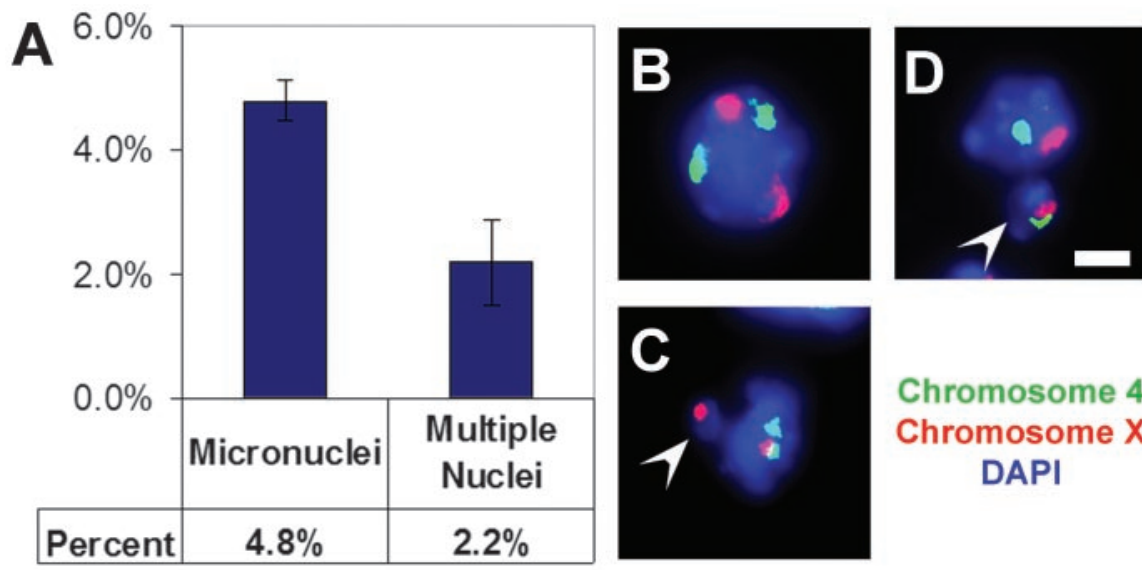

Chromosome 4 Chromosome $\mathrm{X}$ DAPI

Figure 7. NPCs harbor nuclear abnormalities. A, Prevalence of NPC nuclear abnormalities (micronuclei and multiple nuclei) The values represent the average of three independent experiments. At least 220 nestin-positive NPCs pooled from three to five cortices were scored for nuclear abnormalities in each experiment. $B-D$, Dual-color FISH for chromosomes 4 (green) and X (red) on interphase NPCs. Normal female cells contain two copies of each chromosome $(B)$. In a fraction of micronucleated cells, the aneuploid main nucleus is accompanied by a micronucleus (arrowhead) that is positively labeled for either chromosome $(C)$ or both chromosomes $(D)$ examined. Nuclei are counterstained with DAPI. Scale bar, $5 \mu \mathrm{m}$. onic and neural stem cells, aneuploidy may also influence these less-differentiated stem cells by similar mechanisms. Indeed, spontaneous aneuploidy, most likely resulting from mitotic nondisjunction, has been reported in mouse embryonic stem cells (Cervantes et al., 2002) and during early human postzygotic development (Kalousek, 2000). Although the precise function of neural aneuploidy is not yet known, aneuploidy can clearly mod- 

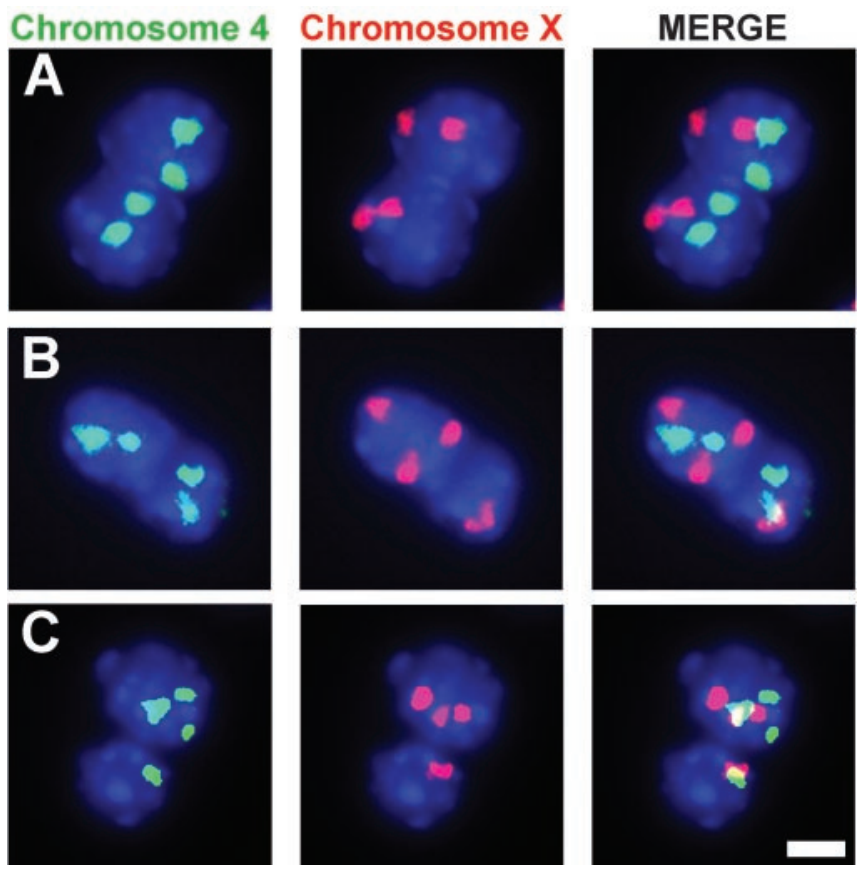

Figure 8. Nondisjunction occurs in NPCs. A, Equal segregation of chromosomes 4 and X. This mitotic NPC is blocked in cytokinesis by cytochalasin B treatment and becomes binucleate. Dual-color FISH reveals a 2:2 distribution of hybridization signals for both chromosomes 4 (green) and X (red). $B$, Nondisjunction of chromosome X. This cytokinesis-blocked binucleate NPC shows a 3:1 distribution of hybridization signals for chromosome X. C, Nondisjunction of chromosomes 4 and X. This cytokinesisblocked binucleate NPC shows a 3:1 distribution of hybridization signals for both chromosomes 4 and X. Nuclei are counterstained with DAPI (blue). Scale bar, $5 \mu \mathrm{m}$.

ulate global gene expression in cells (Hughes et al., 2000; FitzPatrick et al., 2002; Kaushal et al., 2003). Moreover, the mosaicism produced by the intermingling of euploid and genotypically diverse aneuploid cells could contribute to the fine organization of the developing and mature nervous system.

\section{References}

Adams RJ (1996) Metaphase spindles rotate in the neuroepithelium of rat cerebral cortex. J Neurosci 16:7610-7618.

Allen DM, van Praag H, Ray J, Weaver Z, Winrow CJ, Carter TA, Braquet R, Harrington E, Ried T, Brown KD, Gage FH, Barlow C (2001) Ataxia telangiectasia mutated is essential during adult neurogenesis. Genes Dev 15:554-566.

Barch MJ, Knutsen T, Spurbeck JL (1997) The AGT cytogenetics laboratory manual. Philadelphia: Lippincott-Raven.

Barnes DE, Stamp G, Rosewell I, Denzel A, Lindahl T (1998) Targeted disruption of the gene encoding DNA ligase IV leads to lethality in embryonic mice. Curr Biol 8:1395-1398.

Bergoglio V, Pillaire MJ, Lacroix-Triki M, Raynaud-Messina B, Canitrot Y, Bieth A, Gares M, Wright M, Delsol G, Loeb LA, Cazaux C, Hoffmann JS (2002) Deregulated DNA polymerase beta induces chromosome instability and tumorigenesis. Cancer Res 62:3511-3514.

Bhattacharjee MB, Armstrong DD, Vogel H, Cooley LD (1997) Cytogenetic analysis of 120 primary pediatric brain tumors and literature review. Cancer Genet Cytogenet 97:39-53.

Blaschke AJ, Staley K, Chun J (1996) Widespread programmed cell death in proliferative and postmitotic regions of the fetal cerebral cortex. Development 122:1165-1174.

Brinkley BR (2001) Managing the centrosome numbers game: from chaos to stability in cancer cell division. Trends Cell Biol 11:18-21.

Cahill DP, Kinzler KW, Vogelstein B, Lengauer C (1999) Genetic instability and darwinian selection in tumours. Trends Cell Biol 9:M57-M60.

Cervantes RB, Stringer JR, Shao C, Tischfield JA, Stambrook PJ (2002) Embryonic stem cells and somatic cells differ in mutation frequency and type. Proc Natl Acad Sci USA 99:3586-3590.
Chenn A, Zhang YA, Chang BT, McConnell SK (1998) Intrinsic polarity of mammalian neuroepithelial cells. Mol Cell Neurosci 11:183-193.

Chun J, Jaenisch R (1996) Clonal cell lines produced by infection of neocortical neuroblasts using multiple oncogenes transduced by retroviruses. Mol Cell Neurosci 7:304-321.

Chun J, Schatz DG (1999) Rearranging views on neurogenesis: neuronal death in the absence of DNA end-joining proteins. Neuron 22:7-10.

Deans B, Griffin CS, Maconochie M, Thacker J (2000) Xrcc2 is required for genetic stability, embryonic neurogenesis and viability in mice. EMBO J 19:6675-6685.

Difilippantonio MJ, Zhu J, Chen HT, Meffre E, Nussenzweig MC, Max EE, Ried T, Nussenzweig A (2000) DNA repair protein Ku80 suppresses chromosomal aberrations and malignant transformation. Nature 404:510-514

Doxsey SJ, Stein P, Evans L, Calarco PD, Kirschner M (1994) Pericentrin, a highly conserved centrosome protein involved in microtubule organization. Cell 76:639-650.

Fenech M (2000) The in vitro micronucleus technique. Mutat Res 455:81-95.

FitzPatrick DR, Ramsay J, McGill NI, Shade M, Carothers AD, Hastie ND (2002) Transcriptome analysis of human autosomal trisomy. Hum Mol Genet 11:3249-3256.

Frank KM, Sharpless NE, Gao Y, Sekiguchi JM, Ferguson DO, Zhu C, Manis JP, Horner J, DePinho RA, Alt FW (2000) DNA ligase IV deficiency in mice leads to defective neurogenesis and embryonic lethality via the p53 pathway. Mol Cell 5:993-1002.

Fukushima N, Weiner JA, Kaushal D, Contos JJ, Rehen SK, Kingsbury MA, Kim KY, Chun J (2002) Lysophosphatidic acid influences the morphology and motility of young, postmitotic cortical neurons. Mol Cell Neurosci 20:271-282.

Gao Y, Sun Y, Frank KM, Dikkes P, Fujiwara Y, Seidl KJ, Sekiguchi JM, Rathbun GA, Swat W, Wang J, Bronson RT, Malynn BA, Bryans M, Zhu C, Chaudhuri J, Davidson L, Ferrini R, Stamato T, Orkin SH, Greenberg ME, Alt FW (1998) A critical role for DNA end-joining proteins in both lymphogenesis and neurogenesis. Cell 95:891-902.

Gao Y, Ferguson DO, Xie W, Manis JP, Sekiguchi J, Frank KM, Chaudhuri J, Horner J, DePinho RA, Alt FW (2000) Interplay of p53 and DNA-repair protein XRCC4 in tumorigenesis, genomic stability and development. Nature 404:897-900.

Ghadimi BM, Sackett DL, Difilippantonio MJ, Schrock E, Neumann T, Jauho A, Auer G, Ried T (2000) Centrosome amplification and instability occurs exclusively in aneuploid, but not in diploid colorectal cancer cell lines, and correlates with numerical chromosomal aberrations. Genes Chromosomes Cancer 27:183-190.

Griffin CS, Simpson PJ, Wilson CR, Thacker J (2000) Mammalian recombination-repair genes XRCC2 and XRCC 3 promote correct chromosome segregation. Nat Cell Biol 2:757-761.

Gu Y, Sekiguchi J, Gao Y, Dikkes P, Frank K, Ferguson D, Hasty P, Chun J, Alt FW (2000) Defective embryonic neurogenesis in Ku-deficient but not DNA-dependent protein kinase catalytic subunit-deficient mice. Proc Natl Acad Sci USA 97:2668-2673.

Haydar TF, Ang Jr E, Rakic P (2003) Mitotic spindle rotation and mode of cell division in the developing telencephalon. Proc Natl Acad Sci USA 100:2890-2895.

Hendzel MJ, Wei Y, Mancini MA, Van Hooser A, Ranalli T, Brinkley BR, Bazett-Jones DP, Allis CD (1997) Mitosis-specific phosphorylation of histone $\mathrm{H} 3$ initiates primarily within pericentromeric heterochromatin during G2 and spreads in an ordered fashion coincident with mitotic chromosome condensation. Chromosoma 106:348-360.

Hughes TR, Roberts CJ, Dai H, Jones AR, Meyer MR, Slade D, Burchard J, Dow S, Ward TR, Kidd MJ, Friend SH, Marton MJ (2000) Widespread aneuploidy revealed by DNA microarray expression profiling. Nat Genet 25:333-337.

Kalousek DK (2000) Pathogenesis of chromosomal mosaicism and its effect on early human development. Am J Med Genet 91:39-45.

Kamei Y, Inagaki N, Nishizawa M, Tsutsumi O, Taketani Y, Inagaki M (1998) Visualization of mitotic radial glial lineage cells in the developing rat brain by Cdc2 kinase-phosphorylated vimentin. Glia 23:191-199.

Kaushal D, Contos JJ, Treuner K, Yang AH, Kingsbury MA, Rehen SK, McConnell MJ, Okabe M, Barlow C, Chun J (2003) Alteration of gene expression by chromosome loss in the postnatal mouse brain. J Neurosci 23:5599-5606. 
Khanna KK, Jackson SP (2001) DNA double-strand breaks: signaling, repair and the cancer connection. Nat Genet 27:247-254.

Kirsch-Volders M, Vanhauwaert A, De Boeck M, Decordier I (2002) Importance of detecting numerical versus structural chromosome aberrations. Mutat Res 504:137-148.

Kuida K, Zheng TS, Na S, Kuan C, Yang D, Karasuyama H, Rakic P, Flavell RA (1996) Decreased apoptosis in the brain and premature lethality in CPP32-deficient mice. Nature 384:368-372.

Lee Y, McKinnon PJ (2002) DNA ligase IV suppresses medulloblastoma formation. Cancer Res 62:6395-6399.

Lengauer C, Kinzler KW, Vogelstein B (1998) Genetic instabilities in human cancers. Nature 396:643-649.

Levine DS, Sanchez CA, Rabinovitch PS, Reid BJ (1991) Formation of the tetraploid intermediate is associated with the development of cells with more than four centrioles in the elastase-simian virus 40 tumor antigen transgenic mouse model of pancreatic cancer. Proc Natl Acad Sci USA 88:6427-6431.

Martinou JC, Dubois-Dauphin M, Staple JK, Rodriguez I, Frankowski H, Missotten M, Albertini P, Talabot D, Catsicas S, Pietra C, Huarte J (1994) Overexpression of BCL-2 in transgenic mice protects neurons from naturally occurring cell death and experimental ischemia. Neuron 13:1017-1030.

Meininger V, Binet S (1988) Spatial organization of microtubules in various types of cells in the embryonic tectal plate of mouse using immunofluorescence after PEG embedding. Biol Cell 64:301-308.

Noctor SC, Flint AC, Weissman TA, Wong WS, Clinton BK, Kriegstein AR (2002) Dividing precursor cells of the embryonic cortical ventricular zone have morphological and molecular characteristics of radial glia. J Neurosci 22:3161-3173.

Pihan GA, Doxsey SJ (1999) The mitotic machinery as a source of genetic instability in cancer. Semin Cancer Biol 9:289-302.

Pihan GA, Purohit A, Wallace J, Malhotra R, Liotta L, Doxsey SJ (2001) Centrosome defects can account for cellular and genetic changes that characterize prostate cancer progression. Cancer Res 61:2212-2219.

Pompeiano M, Blaschke AJ, Flavell RA, Srinivasan A, Chun J (2000) De- creased apoptosis in proliferative and postmitotic regions of the caspase 3-deficient embryonic central nervous system. J Comp Neurol 423:1-12.

Rehen SK, McConnell MJ, Kaushal D, Kingsbury MA, Yang AH, Chun J (2001) Chromosomal variation in neurons of the developing and adult mammalian nervous system. Proc Natl Acad Sci USA 98:13361-13366.

Saavedra HI, Fukasawa K, Conn CW, Stambrook PJ (1999) MAPK mediates RAS-induced chromosome instability. J Biol Chem 274:38083-38090.

Sah VP, Attardi LD, Mulligan GJ, Williams BO, Bronson RT, Jacks T (1995) A subset of p53-deficient embryos exhibit exencephaly. Nat Genet 10:175-180.

Saunders WS, Shuster M, Huang X, Gharaibeh B, Enyenihi AH, Petersen I, Gollin SM (2000) Chromosomal instability and cytoskeletal defects in oral cancer cells. Proc Natl Acad Sci USA 97:303-308.

Seymour RM, Berry M (1975) Scanning and transmission electron microscope studies of interkinetic nuclear migration in the cerebral vesicles of the rat. J Comp Neurol 160:105-125.

Sharpless NE, Ferguson DO, O’Hagan RC, Castrillon DH, Lee C, Farazi PA, Alson S, Fleming J, Morton CC, Frank K, Chin L, Alt FW, DePinho RA (2001) Impaired nonhomologous end-joining provokes soft tissue sarcomas harboring chromosomal translocations, amplifications, and deletions. Mol Cell 8:1187-1196.

Stearns T, Evans L, Kirschner M (1991) Gamma-tubulin is a highly conserved component of the centrosome. Cell 65:825-836.

Sugo N, Aratani Y, Nagashima Y, Kubota Y, Koyama H (2000) Neonatal lethality with abnormal neurogenesis in mice deficient in DNA polymerase beta. EMBO J 19:1397-1404.

Svendsen CN, ter Borg MG, Armstrong RJ, Rosser AE, Chandran S, Ostenfeld T, Caldwell MA (1998) A new method for the rapid and long term growth of human neural precursor cells. J Neurosci Methods 85:141-152.

Weber RG, Bridger JM, Benner A, Weisenberger D, Ehemann V, Reifenberger G, Lichter P (1998) Centrosome amplification as a possible mechanism for numerical chromosome aberrations in cerebral primitive neuroectodermal tumors with TP53 mutations. Cytogenet Cell Genet 83:266-269.

Zheng Y, Jung MK, Oakley BR (1991) Gamma-tubulin is present in Drosophila melanogaster and Homo sapiens and is associated with the centrosome. Cell 65:817-823. 